\title{
Un nuevo diseño para el análisis de la respuesta gráfica al Test de Persona Bajo la Lluvia, aplicado en psicología laboral: detección de los recursos frente al trauma
}

\section{A new design for the analysis of the graphic response to the Person In the Rain Test, applied in labour psychology: detecting the resourses to face trauma}

\author{
Silvina Perez Zambón* \\ Universidad de Ciencias Empresariales y Sociales, Argentina \\ (Rec.: marzo de 2016 - Acept.: septiembre de 2016)
}

\begin{abstract}
Resumen
El propósito del presente trabajo consiste en presentar una nueva propuesta de interpretación de la respuesta gráfica al Test de Persona Bajo la Lluvia (PBLL). Ésta se basa en un diseño de investigación con procedimientos específicos para operacionalizar el concepto de recursos frente al trauma, representado por la situación estresante planteada por la consigna de la técnica proyectiva. Se apuntará a la revisión de conceptos teóricos con el análisis empírico de indicadores presentes en las técnicas gráficas correspondientes a casos de psicología laboral.

Primero definiremos los conceptos centrales (defensas y vivencia de desamparo). Posteriormente, los articularemos con el test de PBLL y presentaremos la operacionalización. Finalmente, realizaremos la discusión de las conclusiones obtenidas.
\end{abstract}

Palabras clave: PBLL, recursos, trauma, vivencia de desamparo, operacionalización, psicología laboral.

\begin{abstract}
The purpose of the present paper is to advance a new proposal for the interpretation of graphic responses to the Draw a Person in the Rain Test (DAPR). This proposal is based on a research design with specific procedures that will enable us to operationalize the concept of resources to face trauma, which is represented by a stressful situation posed by the instructions of the projective technique. We will associate the review of theoretical concepts with the empirical analysis of indicators found in graphic techniques related to cases from the field of work psychology.

First, we will define the main concepts (defenses and helplessness experience). Then, we will articulate these concepts with the DAPR test and present the operationalization. Finally, we will discuss the conclusions reached.
\end{abstract}

Keywords: PIR, resourses, trauma, helplessnss experience, operationalization, labour psychology.

\footnotetext{
* Correspondencia a: Silvina Perez Zambón. Universidad de Ciencias Empresariales y Sociales, Facultad de Psicología, Paraguay 1401, Buenos Aires, Argentina. E-mail: pz.silvina@outlook.com
} 


\section{Introducción}

El campo de las técnicas proyectivas es muy vasto y ha dado lugar a propuestas muy distintas, algunas de las cuales ponen énfasis en la percepción, como el Rorschach, considerado uno de los primeros instrumentos proyectivos, hoy en día vigente. Existen otro tipo de técnicas proyectivas que no están basadas estrictamente en la percepción, sino en la producción de percepciones, imágenes. Las técnicas gráficas se ubican dentro de este conjunto.

Entre las numerosas técnicas gráficas existentes (DFH, HTP, Dibujo Libre, Familia Kinética, etc.), el test de Persona Bajo la Lluvia (PBLL) es una herramienta ampliamente utilizada en numerosos ámbitos, tales como la psicología forense, laboral, educación, entre otros. Se implementa en una batería psicodiagnóstica especialmente en selección de personal, en la que se incluyen entrevistas y otros instrumentos, tanto proyectivos como psicométricos, apuntando a obtener un resultado global, intertest, acerca del carácter de apto o no apto de un postulante a un puesto laboral. Evalúa la imagen de sí mismo que presenta el individuo en condiciones desfavorables. Se presenta como una situación conflictiva ante la cual el sujeto debe reaccionar apelando a los recursos que posee (Perez Zambón, 2014, 2015).

En esta oportunidad presentamos una nueva propuesta de abordaje de la respuesta al test de PBLL que apunta a la operacionalización de la escena representada en el mismo. Nos referimos al concepto de recursos frente al trauma. Este tipo de análisis puede resultar útil en todas las ramas de la psicología en que se aplica la técnica.

En principio, presentamos un marco teórico con los conceptos que guían esta investigación. Luego realizamos un resumen del estado del arte. Posteriormente, presentamos la operacionalización antes mencionada e introducimos algunos indicadores gráficos. Finalmente, realizamos una discusión y conclusión acerca de lo investigado.

\section{Marco teórico}

Dos conceptos resultan centrales a la hora de desarroIlar esta propuesta: por un lado, el concepto de defensa (y en especial la defensa acorde a fines) y, por otro, el de desamparo. Ambas conceptualizaciones han sido desarrolladas por Freud $(1915,1926)$ y retomadas por autores posteriores.

\section{Defensas}

Freud (1915) consideraba a las defensas como destinos o vicisitudes de las pulsiones y, por ende, de los deseos en la vida psíquica. Son formas que desarrolla el yo para enfrentar los conflictos entre tres sectores en pugna: las pulsiones y deseos, la realidad y el superyó. Es así que una forma de categorizar las defensas consiste en identificar de qué lado se coloca el yo y a qué se opone. Las defensas a su vez pueden distinguirse en funcionales (cuando posibilitan una creciente diferenciación y complejización de los procesos internos) o patológicas (cuando conducen al empobrecimiento e indiferenciación)

Los autores recientes concuerdan en que la defensa es un concepto central en todo tipo de estudios. Algunos pusieron el foco en las defensas adaptativas (Rapaport, 1953) y la creatividad (Kris, 1952), mientras que otros como Lacan $(1997,1958)$ se centraron en la importancia de la desestimación de la realidad y la instancia paterna en las psicosis, o, como McDougall (1989), en la desestimación del afecto en las perturbaciones psicosomáticas y las adicciones.

Maldavsky (2004; Maldavsky et al., 2007) desarrolla una categorización de las defensas combinando conceptualizaciones freudianas con un matiz diferencial; considera los siguientes parámetros: 1) Diferenciar las defensas opuestas al deseo, opuestas a la realidad y el superyó, y aquellas que concilian los sectores en pugna. 2) Distinguir entre defensas funcionales y patológicas, siendo las defensas más patológicas cuanto mayor es el costo psíquico del yo para mantenerla, en el sentido de conducir a estados de creciente indiferenciación y descomplejización (Maldavsky, 2013). Esta diferenciación entre funcionales y patológicas aplica tanto a defensas opuestas a la realidad y al superyó como a las opuestas al deseo. 3) Incluye un tercer parámetro consistente en diferenciar defensas centrales (definitorias de las bases de una organización psíquica) y defensas secundarias (contribuyen al trabajo de la defensa central y le imprimen un sello más específico), sean éstas funcionales o patológicas.

Se distinguen cuatro defensas centrales entre las funcionales, por orden decreciente en cuanto al grado de conciliación entre los sectores en pugna: 1) acorde a fines, 2) inhibición, 3) creatividad, 4) sublimación. Entre las defensas patológicas, en el mismo orden decreciente, se distinguen cinco de ellas que son centrales: 1) represión, 2) represión con rasgos caracterológicos, 3) desmentida, 4) desestimación de la realidad y la instancia paterna, 5) desestimación del afecto. Freud afirmaba que todas las defensas se caracterizan por la tentativa de desalojar algo (un juicio, una percepción, un pensamiento, un recuerdo) de la vida anímica y remplazarlo por algo distinto considerado "formación sustitutiva". Por esto, se puede diferenciar a las defensas teniendo en cuenta aquello que el yo pretende desalojar de sí en cada ocasión (lo cual queda desinvestido en la vida psíquica) y aquello a lo que recurre como remplazo de lo rechazado (que queda sobreinvestido).

Podemos decir, entonces, que la defensa acorde a fi- 
Un nuevo diseño para el análisis de la respuesta gráfica al Test de Persona Bajo la Lluvia, APLICADO EN PSICOLOGÍA LABORAL: DETECCIÓN DE LOS RECURSOS FRENTE AL TRAUMA

nes es la de menor costo para el aparato psíquico, es una defensa central, no patológica, que permite el mayor grado de conciliación entre el deseo, la realidad y el superyó.

\section{Vivencia de desamparo}

Encontramos el término desamparo (Perez Zambón, 2014) a lo largo de la obra de Freud, sobre todo en Inhibición, síntoma y angustia (Freud, 1926), bajo el nombre de desvalimiento. Existen dos tipos de desamparo yoico, y dos tipos de angustia (Freud, 1926), uno ante la realidad externa (angustia real) y otro ante la pulsión (angustia neurótica). Freud (1926) afirmaba que lo central de la situación de peligro consiste en la medición de nuestras fuerzas en comparación con su magnitud, es decir, la apreciación de nuestro desvalimiento frente a él. Este desvalimiento puede ser material en el caso del peligro real y psíquico en el caso del peligro pulsional. Entonces define la situación traumática como una escena de desvalimiento vivenciada y la distingue de la situación de peligro.

Ambos tipos de peligro conducen a desarrollar dos reacciones: una afectiva consistente en el estallido de angustia y otra correspondiente a la acción protectora. Freud (1926) decía que puede existir una cooperación acorde a fines entre ambas reacciones, donde una da señal a la otra. Aunque también se puede dar una respuesta inadecuada al fin, consistente en el surgimiento de la parálisis por angustia, en que una se extiende a expensas de la otra.

\section{Estado del arte}

Son varios los autores que han desarrollado propuestas para el estudio de los dibujos y otras producciones gráficas que han constituido extensas publicaciones en libros a lo largo de los años (Goodenough, 1983; Hammer, 1969; Koppitz, 1975; Machover, 1949; Querol \& Chaves Paz, 1997; Querol, \& Alcañiz, 2001). Nos hemos preguntado si entre esos textos alguno ha considerado los conceptos psicoanalíticos centrales, tales como el deseo y la defensa (y su estado). Los textos de Grassano (1977) y de Siquier de Ocampo \& García Arzeno (1974) son especialmente valiosos, ya que las autoras pusieron énfasis en el concepto de defensa, aportando un repertorio de orientación kleiniana. En este enfoque combinan los conceptos kleinianos de las defensas esquizoides y maníacas con los freudianos, de los cuales tomaron en cuenta principalmente las defensas correspondientes a las patologías neuróticas (por ejemplo, anulación y aislamiento en las neurosis obsesivas). Entre los procesos psíquicos que toman en cuenta figuran la creatividad y la sublimación. La propuesta de Grassano combina esta exposición con los desarrollos de D. Liberman (1972) sobre estilos comunicacionales. El hecho de recurrir a los desarrollos referidos a los estilos comunicacionales constituye un acercamiento hacia la realización de una operacionalización de los conceptos. Esta propuesta se constituyó en un aporte original al combinar los estudios con tres instrumentos (el TRO, el Cuestionario Desiderativo y el HTP) para el diagnóstico de diferentes organizaciones psicopatológicas.

Otros autores que afirman que se basan en conceptos psicoanalíticos tienen un enfoque psicopatológico que posee rasgos poco precisos (Maldavsky, 2014). Esto se evidencia en su insistencia en vincular determinados rasgos de los dibujos con alguna organización psicopatológica como esquizofrenia o psicopatía, aunque estas no están claramente definidas en sus aspectos específicos. En otras situaciones, los métodos proponen una relación entre ciertos rasgos y algún deseo, apetito o inclinación interna, o alguna defensa. En los instrumentos para el análisis del material gráfico vigentes las referencias a los conceptos están poco desarrolladas y sus conceptos no son plausibles de ser operacionalizados. Por lo tanto, aunque el texto de Grassano resulta un aporte importante en esta línea, no se han encontrado instrumentos y procedimientos que permitan detectar los deseos en las manifestaciones visuales $y$, menos aún, la combinatoria entre deseos y defensas. Solo algunos libros aportan sugerencias útiles, aunque parciales.

Existe mucha bibliografía respecto de otras técnicas proyectivas. No nos detendremos a citarlas en esta investigación, ya que no constituyen el núcleo fundamental de esta propuesta. En cuanto al test de PBLL, el mismo fue creado sin un marco teórico (no posee categorías propias para el análisis) por lo cual su interpretación se ha ido nutriendo de otras técnicas similares.

Numerosos autores pusieron el foco en los dibujos de los entrevistados (Goodenough, 1983; Hammer, 1969; Koppitz, 1975; Machover, 1949; Querol \& Chaves Paz, 1997) y desarrollaron estandarizaciones de las categorías gráficas que, aunque interesantes y útiles, no permiten establecer generalizaciones. Como explica Maldavsky (2014a) en uno de sus trabajos más recientes respecto del signo visual, en varias técnicas gráficas los autores han encontrado dificultades para estudiar el aporte de la imagen. El test DFH (Goodenough, 1983), por ejemplo, fue creado para analizar los aspectos subjetivos (como test proyectivo), así como el desarrollo intelectual de un entrevistado. Como test proyectivo, se observó en sus versiones iniciales un optimismo a-crítico (Machover, 1949) acerca de la capacidad del instrumento para detectar deseos, defensas y rasgos de patología esquizofrénica, etc. Posteriormente, fue revisado por Portuondo (1973), quien detalló algunos criterios adicionales de interpretación algo más precisos y aseguró que convenía articular este test con la aplicación de otros instrumentos o entrevistas y con el empleo del enfoque psicoanalítico. Casullo (1998) alcanzó mayor éxito en su intento de regular el DFH para 
la investigación del desarrollo intelectual, lo cual derivó en el desarrollo de baremos regionales argentinos.

Se han encontrado estudios que apuntan a detectar determinados deseos y defensas en materiales gráficos (Sneiderman 1998, Nudel 2014), algunos de ellos aplicando la metodología que se pretende emplear en esta investigación, pero ninguna al test de PBLL.

La ausencia de baremos específicos hace difícil la interpretación de los materiales gráficos correspondientes al test de PBLL y reduce su grado de confiabilidad y validez. Para paliar esta dificultad, otros autores han combinado el test de PBLL con el empleo de otras técnicas (Borelle, Maida \& De Luca, 2013; Burin, Jimenez \& Meler, 2007; Carney, 1992; de Souza \& Guerrero, 2005; Fatelevich et al., 2008; Piccone \& Passalacqua, 2010; Pool, 2006; Prichard, 1998; Rossi, 1997; Taylor, 1977; Verinis, Lichtenberg \& Henrich, 1974; Willis, Joy \& Kaiser, 2010).

No se han encontrado investigaciones sobre otras técnicas proyectivas que permitan estudiar el desvalimiento poniendo el foco en el método de análisis.

\section{Métodología}

\section{Muestra}

El campo sobre el cual se investigó consistió en las respuestas al Test de PBLL de 500 postulantes a ingreso a un mismo puesto de trabajo. Edad: entre 17 y 25 años, sexo: $80 \%$ hombres y $20 \%$ mujeres, clase social media-baja y nivel educacional, en su mayoría, de primario completo. Resulta una muestra bastante homogénea, que puede permitir realizar estudios comparativos poblacionales. A su vez, estos casos pueden ser representativos de un conjunto importante en Argentina, que son aquellos sujetos que buscan su primer trabajo.

\section{Procedimiento}

Los casos fueron tomados de una base de datos correspondiente a la institución donde la autora se desempeña como psicóloga. Corresponden a procesos de selección realizados en 2012, de manera grupal (de aproximadamente 30 evaluados), a cargo de diferentes profesionales. Por esto, no se cuenta con información de los sujetos respecto de co-morbilidades con patologías psiquiátricas, antecedentes laborales o familiares.

Técnicas e instrumentos: test de $P B L L, A D L$

\section{Técnicas proyectivas}

Según Caride (1999), los métodos proyectivos se desarrollan a partir de la necesidad de contemplar las diferencias individuales y el intento de dar un enfoque científico al estudio de esas discrepancias, considerando aspectos más complejos de la personalidad. Los métodos existentes hasta el momento no cubrían esa necesidad, ya que se fundamentaban en teorías de enfoque atomístico (Caride, 1999).

Los instrumentos proyectivos constituyen un grupo de procedimientos (Caride, 1999) que, recurriendo a estímulos ambiguos y con distinto grado de estructuración, suscitan en los entrevistados respuestas libres, que informan respecto de aspectos de su personalidad, tales como sus conflictos, sentimientos y tendencias.

Las técnicas gráficas son instrumentos proyectivos que se utilizan en el proceso psicodiagnóstico a fin de explorar aquellos aspectos más arcaicos y primitivos del sujeto, remitiendo a niveles básicos de organización e integración de la subjetividad. El test de PBLL se ubica dentro de este conjunto, presentándose como una situación conflictiva ante la cual el sujeto debe reaccionar, apelando a los recursos que posee.

\section{El algoritmo David Liberman}

El algoritmo David Liberman (ADL) (Maldavsky, 2013) es un método de análisis de las manifestaciones discursivas que permite detectar deseos, defensas y estados en el discurso de los hablantes. Cuenta con instrumentos específicos para cada nivel de análisis: grillas e instrucciones para el estudio de los relatos (ADL-R) y de los actos del habla (ADL-AH), así como un diccionario computarizado para el análisis de las palabras (ADL-P). En cuanto a la imagen, presenta un instrumento para el estudio del signo visual (ADL-SV). En su vertiente icónica, que se refiere a la imagen como relato, el instrumento se vale de las escenas (acciones o estados), los complementos de tales escenas (objetos, ayudantes, atributos), los desempeños motrices y las distancias en las imágenes (Maldavsky, 2014a, 2014b).

\section{Resultados}

Operacionalización del concepto de defensa y de la escena de desamparo en el test de PBLL

Sintetizando el texto de Freud (1926), podemos decir que ante una situación de peligro se suelen dar dos tipos de reacciones, una afectiva consistente en el estallido de angustia (señal), y otra motora, consistente en la acción protectora. Esto sería el proceso acorde a fines. El desamparo surge cuando no se da esa respuesta acorde a fines, y en su lugar surge una parálisis (crisis) de angustia que imposibilita el acceso a la acción protectora. Entonces se despliega un tipo de respuesta que puede llegar a ser traumática.

Teniendo en cuenta estos conceptos, analizaremos lo que ocurre con el test de PBLL. La consigna solicita al sujeto evaluado que dibuje una persona bajo la lluvia. Lo particular de este test consiste en que la situación planteada por la consigna somete al evaluado a la vivencia de desamparo ante una realidad que resulta de carácter estresante, exigente. Es decir, el test de PBLL 
permite detectar si se ha dado una buena implementación de los recursos para encarar dicho escenario, lo cual nos puede llevar a inferir si hubo o no angustia señal, de carácter apropiado. Mediante la operacionalización de esta escena podemos medir el grado de desamparo que presenta un individuo ante una situación de peligro proveniente del exterior, la cual va desde situaciones traumáticas, pasando por estados de inadecuación parcial, hasta respuestas acorde a fines. Cabe aclarar que lo que nos interesa operacionalizar no es la vivencia de desamparo o la escena estresante o el trauma, sino más bien la respuesta ante ella, la cual puede llegar a ser traumática. Esta operacionalización implica que nos preguntemos cuáles son los recursos implementados por el sujeto. El concepto de recursos se ve representado por las defensas funcionales (acordes a fines) exitosas a las que apelará el individuo. Al mismo tiempo, podremos observar las dificultades surgidas en este punto, las cuales quedarán representadas por las defensas funcionales fracasadas o por las defensas patológicas, en especial estas últimas. Podemos realizar este análisis de las defensas y su estado mediante los conceptos e instrumentos del ADL (Maldavsky, 2004, 2014a, 2014b; Maldavsky et al, 2007, 2013).

El paso siguiente consiste en definir en qué partes de la respuesta gráfica se detectan estas cuestiones. Para ello, primero debemos categorizar la respuesta gráfica. Dentro de la categoría de dibujo, hay dos grandes sub-categorías (Maldavsky, 2013, 2014a, 2014b): el componente icónico (escena representada en la imagen, imagen como relato) y el plástico (forma en que fue graficada esa imagen). Otros autores (Caride, 2008) mencionan el signo icónico cuando hablan del análisis del contenido de lo graficado y proponen considerar la categoría de los objetos seleccionados dentro de una temática, el tratamiento que se hace del dibujo (si es que omiten, agregan, tachan, borran, remarcan, etc.), el simbolismo, la relación de lo graficado con la realidad consensual, el cromatismo y el sombreado. En el caso del PBLL, las partes centrales del signo icónico corresponden al dibujo de la persona, de la condición climática y del elemento protector. Las dos primeras son solicitadas de manera explícita en la consigna, mientras que la última resulta la de mayor carácter proyectivo, pues es lo que se espera que el sujeto logre manifestar. El elemento protector constituye el nexo entre la figura humana y la condición climática. Se pueden detectar los recursos frente al trauma (representado por la situación estresante) mediante el análisis de la existencia o no del elemento de protección y el modo en que ha sido empleado. Las características de la figura humana no constituyen indicadores de la defensa central frente al trauma, sino que más bien refieren a la personalidad global. Los rasgos de la condición climática permiten observar el tipo de trauma y su magnitud. Estos dos componentes cumplen una función comple- mentaria, pues el logro de la protección depende en cierta medida de sus características.

En cuanto a los componentes del nivel plástico de la imagen, Caride (2008), al hablar del dibujo de figura humana, los agrupaba en dos categorías: la gráfica y la formal o estructural. Dentro de la categoría gráfica se encuentra el tipo de trazo y la presión del mismo. A su vez, dentro del componente formal o estructural ubica: emplazamiento, secuencia, tamaño, movimiento y expresión, perspectiva.

Al respecto es válido hacer una aclaración más. Al tratarse de una respuesta gráfica, es posible detectar numerosas cuestiones. Además de puntualizar si la defensa es funcional o no, se pueden observar otros rasgos de los recursos que constituyen los estilos en que un sujeto se defiende del desamparo producido por la situación traumática, como el hecho de que al ampararse ordena o embellece, entre otros. Algunos de estos elementos que pueden ser detectados derivan de estudios del nivel icónico, y otros del nivel plástico. Estas cuestiones puede que se manifiesten con mayor claridad en algunos gráficos que en otros. No obstante, el objetivo de esta investigación consiste en detectar el recurso central que manifiesta el sujeto ante una situación estresante, es decir, la defensa central en relación con el trauma o con el desamparo. Este recurso puede o no ser acorde a fines, siendo los estilos el complemento de aquella defensa nuclear. Es importante señalar también que el test de PBLL está diseñado para el estudio de la estructura psíquica global del sujeto, aunque ese tema tampoco constituye lo central de esta investigación. La finalidad de este trabajo no consiste en realizar un aporte al diagnóstico en general, sino en el terreno laboral y ligado con la cuestión de hasta dónde un sujeto puede responder ante una situación traumática.

\section{Indicadores gráficos de las defensas frente al trauma}

\section{Componente icónico}

Como dijimos, el elemento del dibujo que nos permite inferir las defensas o recursos de los que hablamos es el elemento protector.

Respecto de este componente del signo icónico, lo más común es encontrar dibujos de paraguas, por lo cual lo consideramos el cliché. Otros tipos de protecciones que aparecen con cierta frecuencia son el piloto, botas de lluvia, capa y sombrero, un periódico sostenido sobre la cabeza, un árbol, un alero, techo de una parada de colectivo. Su sola presencia reviste importancia, ya que no está implícito en la consigna, mas sí en la escena solicitada y en el objetivo de la técnica. Es decir, es el componente gráfico con mayor saturación proyectiva de la técnica.

Por lo tanto, el primer paso en el análisis consiste en precisar si el elemento protector se encuentra presen- 
te o no. Comencemos por analizar la ausencia de elemento protector. En este caso se suele evidenciar dibujos de figuras humanas erguidas siendo alcanzadas por las gotas de lluvia. A simple vista observamos que el sujeto se presenta a merced de las inclemencias del tiempo, es decir, desamparado. Esto nos permite inferir que no ha podido desplegar recursos adecuados para hacer frente a la situación apremiante que ha resultado traumática, recurriendo a defensas patológicas ante el trauma. Éstas pueden tener, a su vez, tres estados: a) exitoso (por ejemplo cuando el sujeto se encuentra desamparado ante la lluvia pero con una expresión de felicidad en el rostro y una actitud de baile, al estilo del clásico "Singing in the rain"); b) fracasado (por ejemplo, figura humana sin paraguas, desvalida ante la lluvia y con el semblante apagado; otros dibujan un sujeto a merced de la lluvia al que solo agregan una capucha) o c) mixto (los rasgos de la figura humana o del contexto permiten inferir que se encuentra realizando una actividad en la que no es común o viable utilizar protección contra la lluvia, por ejemplo, un jugador de fútbol o un hombre sembrando el campo).

Otro grupo lo constituyen aquellos dibujos en que el elemento protector se encuentra presente. En este caso, es posible inferir que la defensa es acorde a fines y se pueden dar tres alternativas que se vinculan con el estado de la misma: (a) exitoso: el elemento protector cumple su función, protege de la lluvia, soporta la magnitud de las inclemencias del tiempo y es sostenido por una figura humana acorde al mismo; (b) fracasado: elemento de protección presente pero de manera tal que no cumple su función protectora, por ejemplo, casos en que el paraguas aparece volando en el aire producto de un fuerte viento, o tirado en el piso, o roto; (c) mixto: hay paraguas pero llueve de costado y el sujeto se termina mojando igual, o se moja los zapatos y el pantalón porque está inundado o hay charcos.

Resulta evidente el criterio que permite diferenciar entre defensas acorde a fines y defensas patológicas, pues esto queda determinado por la presencia o ausencia del elemento protector. El análisis se complejiza a la hora detectar el estado de dichas defensas. En el primer caso, el de las defensas acorde a fines, consideramos que el criterio que resulta útil para diferenciar los estados de dicha defensa es el hecho de que se moje la figura humana o no, o lo haga a medias. Es por esto que la defensa es exitosa si el sujeto está bien cubierto; es fracasada si, a pesar de aparecer la protección en el dibujo no cumple su función; y es mixta si la protección cumple su función a medias, es decir, deja al descubierto alguna parte del cuerpo, en general una o dos extremidades, quedando cubierta la mayor parte de la figura humana. En cuanto a las defensas patológicas, un indicador que nos ha resultado útil en los casos en que los dibujos aparecen desamparados ante la lluvia, es aquel vinculado con la actitud del su- jeto frente a este hecho. Esto puede inferirse en base al análisis de algunos rasgos del signo visual correspondientes, en su mayoría, al contexto y a la figura humana. Respecto de la figura humana, podemos considerar la expresión facial junto con la rigidez del cuerpo y la ausencia de un contexto. Para realizar este análisis podemos valernos del instrumento ADL-M (Maldavsky, 2013), el cual cuenta con grillas que categorizan programas gesticulares y motrices. Es decir, aquellos casos en que se evidencie una especie de desierto en que solo hay una figura humana con lluvia; que a su vez esta figura presente en su expresión facial una risa de tipo "tonta" (expresión vacía, boca representada por una línea en forma de onda, cerrada, en algunos casos con ojos vacíos, sin posibilidad de ser asociada a otras cuestiones del dibujo) y en su cuerpo tenga una actitud rígida (piernas juntas o separadas, brazos pegados al cuerpo, sin indicadores de movimiento o articulación), serán considerados como correspondientes a defensas patológicas exitosas. Esta combinatoria significa que no hay protección ni actitud de protegerse, al igual que ocurre en aquellos casos en que aparece un sujeto a merced de la lluvia, con una sonrisa de boca abierta y una actitud del estilo "Singing in the rain". Un aspecto característico de este conjunto de sujetos consiste en que parecen encontrarse en un estado de parálisis, donde se encuentra desestimado el afecto que se supone asociado a la situación que plantea el test (estrés, angustia, ansiedad), y en su lugar aparece otro afecto o ninguno. Por otro lado, aquellos sujetos que se encuentran desprotegidos de la lluvia, en algunos casos con rasgos corporales de parálisis y en otros con actitud de correr o refugiarse, pero que presentan una actitud de agobio, tristeza, enojo o desagrado, serán asociados a defensas patológicas en estado fracasado. Un grupo intermedio son los que representan defensas patológicas en estado mixto. Pensamos que el indicador que nos permite agrupar estos casos se asocia con aquellos dibujos en que el contexto y los rasgos de la figura humana permiten identificar el tipo de actividad que está realizando, y a su vez, esa actividad suele ser llevada a cabo a pesar de la lluvia, muchas veces sin posibilidad de protección. Son ejemplos comunes de este grupo los dibujos de futbolistas, campesinos, sujetos haciendo guardia o pescando. La defensa sigue siendo patológica porque se moja, y puede que haya un cierto grado de malestar, pero continúan con su proyecto a pesar de la lluvia, ya sea porque se disfruta la actividad o se toma como una obligación.

\section{Otros indicadores}

Hemos dicho que el test de PBLL, al igual que otras técnicas proyectivas gráficas permite realizar numerosos tipos de análisis dependiendo del diseño de investigación que se utilice y los objetivos de la misma. En este caso, hemos optado por considerar sólo el estudio del elemento protector de esta técnica, ya que es el 
Un nuevo diseño para el análisis de la respuesta gráfica al Test de Persona Bajo la Lluvia, APLICADO EN PSICOLOGÍA LABORAL: DETECCIÓN DE LOS RECURSOS FRENTE AL TRAUMA

que mejor nos permite observar los recursos frente a la situación traumática.

A continuación presentamos los indicadores que pueden detectarse en el dibujo de los otros componentes del signo icónico del test de PBLL, es decir la figura humana y las condiciones climáticas. Los mismos no serán considerados en esta investigación pues no hacen a su diseño y sus objetivos. No obstante, pensamos que resulta útil categorizarlos, ya que pueden servir de complemento, además de ser útiles para investigaciones con otros diseños y objetivos, como las que hemos mencionado anteriormente. Agregamos el análisis de algunos componentes plásticos.

Figura humana en el test de PBLL

Basamos el análisis de este componente en las propuestas realizadas por Caride (2008), quien proponía considerar, dentro del estudio del contenido, la categoría de los objetos seleccionados dentro de una temática, el tratamiento que se hace del dibujo (si es que omiten, agregan, tachan, borran, remarcan, etc.), simbolismo, relación de lo graficado con la realidad consensual, cromatismo y sombreado. En base a las características del test de PBLL, hemos decidido considerar las siguientes categorías: la postura, el grado de realidad, su nivel de completud y sus proporciones, la presencia de transparencias, sombreados y dobles. Las mismas pueden indicar el estilo con que se defiende el sujeto.

En cada categoría se puede observar detectar el tipo de defensa operante y su estado. A continuación presentamos un listado de las categorías y el tratamiento que se da a cada una, según estos criterios, mostrando algunos ejemplos respecto de los estados de las defensas. Es conveniente recordar que el análisis de estos componentes dependerá del objetivo y diseño de la investigación que lo enmarque.

Completud: a nivel macro, se considera completa aquella figura humana que presente todos sus miembros y órganos sensoriales visibles. No debe poseer agregados ni faltantes (por ejemplo, un tercer ojo, una pierna menos, un tercer brazo semi borrado o tachado, parte de la cara o la cabeza tapados por el paraguas). Será acorde a fines una figura completa, mientras que la incompletud (por ejemplo, falta de la nariz o la boca, ausencia de una o ambas manos, ausencia de pies o piernas, omisión del cuello) se verá asociada a defensas patológicas.

Acorde a fines: exitosa (presenta todos los miembros y órganos sensoriales adecuadamente dibujados), fracasada (figura humana desnuda o poco abrigada) o mixta (ojos cerrados, cara tapada por el paraguas, mano detrás del tronco o en el bolsillo, ausencia de piernas por perspectiva del dibujo).

Patológicas: exitosas (agregado de brazo extra), fracasadas (ausencia de rostro por omisión, ojos vacíos, sin boca, olvido de dibujar una mano) o mixtas (dedos puntudos, presencia de dientes).

Proporción: el dibujo debe mantener una proporción general y además debe haber proporción entre las partes del mismo. Se espera que los dibujos guarden una simetría realista, aunque suelen observarse alteraciones (por ejemplo, un brazo excesivamente grande en comparación con los otros miembros, edificios más pequeños que la figura humana, entre otros). Será acorde a fines una figura con proporciones adecuadas, mientras que la desproporción se asociará a defensas patológicas.

Acorde a fines: exitosa (adecuada proporción interna y con el contexto), fracasada (inadecuada proporción interna y con el contexto), o mixta (implican proporción interna pero desproporción con el contexto).

Patológicas: exitosas (exageración del tamaño de un miembro), fracasadas (grado extremo de desproporción) o mixtas (combinatoria de desproporciones, donde un miembro es demasiado grande y otro demasiado pequeño).

Postura: puede estar graficado de frente, de perfil o de espalda, siempre y cuando presente una postura erguida. Es lo esperable pues es así como se suele graficar una figura humana. En este indicador se tendrán en cuenta también cuestiones vinculadas con el grado de rigidez o flexibilidad presentado por la figura, para lo cual la motricidad del tronco y brazos junto con la motricidad facial nos aportarán especial información, y en algunos casos también la motricidad de las extremidades inferiores. Será acorde a fines una figura con una postura erguida, mientras que otros tipos de posiciones se verán asociada a defensas patológicas.

Acorde a fines: exitosa (postura erguida, flexible y bien parado), fracasada (postura demasiado rígida) o mixta (equilibrio inestable, como volándose por el viento o en puntas de pie).

Patológicas: exitosas (de espaldas), fracasadas (cabeza abajo, acostado) o mixtas (doblado en la mitad).

Grado de realidad: existen dos alternativas de realidad: que dibuje una realidad concreta o que dibuje la norma. Para precisar esta categoría nos valemos de los desarrollos del grupo de retóricos de Lieja (Groupe $\mu$, 1993), quienes afirmaban que el componente icónico de la imagen corresponde a lo que esta representa del mundo supuesto. Esto se relaciona con el grado en que se asemejan los elementos de la imagen a los vínculos que el sujeto ha construido con la representación de ese mundo exterior supuesto. La representación de ese objeto supuesto puede ser la de un objeto determinado, y también la de un tipo de objeto. La repre- 
sentación de un objeto es a su vez una manifestación de un tipo de objeto, que consiste en una representación mental que el autor de la manifestación tiene de él (Maldavsky, 2014a). Es importante en este caso que grafique una persona y no un personaje, como por ejemplo, un hombre lobo, un superhéroe, un monigote, etc. Se tendrán en cuenta para el análisis de este indicador, agregados y detalles de la vestimenta y el contexto. Será acorde a fines una figura humana con adecuado grado de realidad, mientras que la ausencia de realidad se verá asociada a defensas patológicas.

Acorde a fines: exitosa (realista, con detalles adecuados), fracasada (contorno de figura humana sin relleno, aunque no nos queda claro si esto engloba cierto grado de patología) o mixta (con rasgos infantiles).

Patológicas: exitosas (personaje ficticio en lugar de figura humana), fracasadas (figura de palotes o monigote) o mixtas (cabeza y tronco con extremidades de palotes, palotes rellenados).

Transparencias: las transparencias son aquellos sectores del dibujo que dejan ver objetos que están por detrás. En el caso de la figura humana se pueden observar transparencias respecto del fondo o de alguna prenda de ropa.

Acorde a fines: exitosa (aquellas que remiten a un material o tela transparente en la realidad: bolsa, tul, etc.), fracasada (figuras humanas que dejan ver alguna parte del fondo: línea de base) o mixta (una capa o piloto agregado en transparencia).

Patológicas: exitosas (transparencia de órganos internos), fracasadas (relleno de palotes) o mixtas (no nos queda del todo claro en qué casos se consideraría mixta).

Sombreados: existe un tipo de sombreado que se utiliza para pintar, oscurecer una parte, en el caso de los dibujos en que sólo se cuenta con el lápiz negro. Este tipo de sombreado es el que se suele observar en el cabello de las figuras humanas, y dependiendo de su magnitud, de la comparación con las otras partes del dibujo y la relación con el relato, puede que no se considere problemático. Por otra parte, en general se suele pensar que el sombreado evidencia angustias depresivas, que en este test probablemente se relacione con una falta de recursos para defenderse de la lluvia, es decir, un cierto grado de indefensión. Este tipo de sombreado puede considerarse patológico sobre todo si se evidencia en gran parte o en toda la figura humana.

Acorde a fines: exitosa (sombreado moderado utilizado para pintar, por ejemplo el cabello), fracasada (pintar de manera excesiva, por ejemplo alguna prenda de ropa) o mixtas (no nos queda del todo claro en qué casos se consideraría mixta).
Patológicas: exitosas (no nos queda del todo claro en qué casos se consideraría exitosa), fracasadas (figura humana completamente sombreada) o mixtas (alguna parte del cuerpo sombreada).

\section{Condición climática en el test de PBLL}

Con respecto a las condiciones climáticas, su análisis contribuye más información respecto del tipo específico de desamparo en que se encuentra el sujeto, que de los recursos utilizados. No obstante, la comparación de la magnitud de las condiciones climáticas con los rasgos del elemento protector y de la figura humana puede aportar también algunos matices que enriquezcan el análisis.

Este componente consistirá en cualquier tipo de dibujo que dé el indicio de lluvia (en especial gotas, líneas, puntos, llovizna, nubes). Como hemos mencionado, este ítem será considerado en cuanto a su magnitud respecto del elemento protector, y al igual que la figura humana, también nos aportará algunos matices útiles dependiendo de los objetivos y diseño de la investigación. Por ejemplo, si el paraguas cumple su función por estar colocado sobre la figura humana, pero lo hace a medias ya que resulta un tanto pequeño respecto de la persona y además el sujeto ha graficado una intensa lluvia, entonces podremos inclinar nuestro análisis con mayor facilidad hacia la defensa acorde a fines en estado mixto o fracasado. Lo que suele manifestarse en este punto es la exageración: demasiada lluvia, gotas desproporcionadamente grandes, excesiva cantidad de gotas.

Otro indicador que se observa con cierta frecuencia es la perseveración en gran parte del dibujo, por ejemplo, en el modo de graficar las gotas en series ordenadas. La exagerada cantidad de nubes o la presencia de relámpagos o vientos fuertes (lo cual puede estar hablando de una tormenta más que de una lluvia) no es esperable, pues se espera que logre moderar su ansiedad y controlar la situación. En todo caso, si ha dibujado una tormenta, conviene considerar el grado de protección que ha utilizado y los rasgos de la figura humana. Por lo tanto, consideraremos si es una lluvia acorde al resto del dibujo o si la condición climática es excesiva, tendiendo más hacia lo patológico. Corresponderá a la defensa acorde a fines en estado exitoso la presencia de indicadores de lluvia (gotas, nubes, Ilovizna), manifiestos de manera moderada y en concordancia con las características del elemento protector y de la figura humana. El estado de la defensa será fracasado si la magnitud de dicha condición climática excede al elemento protector y termina mojando a la figura humana. La defensa tendrá estado mixto si la lluvia, a pesar de no caer directamente sobre la figura humana, la termina mojando por ejemplo, por la presencia de charcos o vientos de costado. Por otro lado, si las defensas son patológicas entonces aparecerán 
lluvias demasiado fuertes (de una intensidad mayor a la que podría corresponder a defensas exitosas fracasadas), tormentas ante las cuales el sujeto se encuentra desamparado, por ejemplo, aquellos dibujos en que hay una sola nube con intensa cantidad de lluvia cayendo sólo sobre el sujeto (estado fracasado).

Se pueden encontrar también lluvias o lloviznas que recubren la hoja excepto en el sector donde se encuentra la figura humana (asociadas a defensas patológicas en estado mixto, pues si bien correspondería que el personaje se moje, se evita graficar la lluvia en su sector, por lo cual en el momento actual no es alcanzado por las gotas). Otra opción, como hemos mencionado respecto del elemento protector, son aquellas lluvias que mojan a la figura humana pero a éste no parecen afectarle ni interrumpirle su actividad y de hecho las recibe con una expresión facial de agrado (asociadas con defensas patológicas en estado exitoso).

\section{Componente plástico}

Con respecto al trazo, lo esperable es que sea armónico y firme, lo cual se asocia con la defensa acorde a fines en estado exitoso. Cualquier alteración tendrá que ver con el estado de la defensa (por ejemplo, la defensa acorde a fines en estado fracasado puede manifestarse en trazos esbozados, y en estado mixto, en trazos esbozados sólo en algún sector del dibujo) o con defensas patológicas (por ejemplo, las defensas patológicas en estado fracasado pueden evidenciarse en trazos entrecortados, desintegrados o espigados, y en estado mixto, en trazos temblorosos). En este caso conviene considerar el trazo en el elemento protector, pero a su vez en el dibujo de la figura humana, ya que es allí donde se manifiesta de manera más fácilmente sensible, debido a que en ella se proyecta lo referente a la imagen de sí mismo que posee el sujeto.

En cuanto a la presión, lo esperable es una adecuada presión, ni muy trasparente ni demasiado marcado, en la mayor parte del dibujo, aunque como en los trazos, haciendo hincapié en la figura humana. Este tipo de presión se asocia con la defensa acorde a fines en estado exitoso. Cualquier alteración tendrá que ver con el estado fracasado (por ejemplo, demasiada o demasiado poca presión en la mayor parte del dibujo) o mixto (demasiada o demasiado poca presión en algún sector del dibujo) de esa defensa o con defensas patológicas (las cuales pueden ser, por ejemplo, fracasadas cuando hay excesiva presión que marca la hoja en su reverso o incluso la rompe). Tal vez sea conveniente continuar investigando en esta línea para poder precisar estas categorías con mayor claridad.

\section{Discusión}

Al realizar investigaciones con instrumentos que detectan numerosas cuestiones, se debe elaborar un diseño que permita estudiar una de estas cuestiones y no todas, delimitando de manera precisa el foco del estudio y debiendo organizar los procedimientos de modo tal que sea posible alcanzar ese objetivo. Por lo tanto, pensamos que esta cuestión constituye el aporte más importante de la presente investigación, al contribuir a desarrollar un criterio para estudiar una parte de la enorme riqueza de la manifestación gráfica. Cuando se trata del análisis de producciones gráficas, es necesario ser humilde y recortar. Lo mismo ocurre cuando se tiene por objetivo comparar pinturas de varios periodos, como por ej. "La Virgen con el niño"; en este caso, uno de los elementos que se toma a menudo son los pliegues del manto o el color. Entonces se considera uno de esos rasgos de la pintura y se analizan sus características a lo largo de los distintos periodos correspondientes a la historia del arte. Luego si se quiere realizar un análisis más complejo, se toma otro rasgo, se estudia y se compara con el primero. Lo que queremos decir con esta analogía es que desde el punto de vista metodológico, es conveniente concentrarse en un elemento analizable a la vez, pudiendo luego tomar otros elementos $y$, posteriormente, comparar los análisis entre sí. De lo contrario, puede ocurrir que la investigación se torne demasiado extensa e incluso interminable, o que los resultados terminen siendo de carácter vago o poco preciso.

De todo lo que es posible detectar mediante el dibujo, solo nos interesan aquí determinados indicadores que se vinculan con los conceptos que intentamos operacionalizar. A su vez, el campo en que vamos a aplicar esta operacionalización es el de la psicología laboral, ya que a ese terreno corresponde la muestra y la consigna de la técnica empleada. Este mismo diseño y esta misma consigna pueden servir en el área forense para estudiar abuso, o en el área del apoyo social para analizar neurosis de guerra. Si lo que se quiere es, por ejempo, hacer diagnóstico de personalidad, es conveniente utilizar los mismos instrumentos y realizar otro diseño, tal vez considerando otros indicadores. No obstante, puede ocurrir que al estudiar los casos descubramos algún rasgo de psicosis que, aunque no es lo central en esta investigación, conviene puntualizarlo, ya que ese sujeto puede resultar más vulnerable que otros frente a situaciones complicadas. Por ello, es importante delimitar el foco de manera más estricta para poder avanzar hacia la detección de los indicadores centrales.

El hecho de considerar la presencia o ausencia del elemento de protección, al mismo tiempo que la manera en que ha sido implementado, como indicador del tipo de defensa y su estado frente a la situación traumática resulta una propuesta interesante y novedosa. Este enfoque permite categorizar las respuestas gráficas aprovechando los conceptos freudianos y enriquecer el análisis del test de PBLL mediante la implementación de las conceptualizaciones, categorías e instru- 
mentos del ADL. La mayor riqueza de esta perspectiva radica en que consiste en una categorización de indicadores internos del test de PBLL. Esta no se ha hecho de manera rigurosa hasta el momento, ya que el test en sí mismo carecía de desarrollos teóricos propios y, por lo tanto, los expertos en técnicas proyectivas han ido basando sus análisis en categorías de otras técnicas similares, pero con finalidades distintas, como es por ejemplo, el caso del DFH. Asimismo, este enfoque puede permitirnos determinar el grado de vulnerabilidad de un sujeto, el cual puede establecerse en una escala con distintos gradientes que van desde situaciones de desamparo hasta defensas acordes a fines, pasando por escenarios intermedios.

Esta investigación constituye un avance hacia el desarrollo de instrumentos y procedimientos para evaluar los resultados de esta técnica proyectiva de manera más sistemática, mediante categorías propias del test, favoreciendo el acceso al consenso entre investigadores y usuarios del test. Del mismo modo, también podría aportar al desarrollo de estudios poblacionales de diversa índole.

\section{Referencias}

Borelle, A., Maida, M. \& De Luca, M. (2013). El Test Persona Bajo La Lluvia en niños de edad escolar con trastornos somáticos. Psicología y Psicopedagogía, 12(32), 36-55. Recuperado de http://p3.usal.edu.ar/ index.php/psico/article/view/1892/2445

Burin, M., Jiménez Guzmán, M. \& Meler, I. (Comps.). (2007). Precariedad laboral y crisis de la masculinidad: impacto sobre las relaciones de género. Buenos Aires: UCES.

Caride, M. (1999). "Acerca de las Técnicas proyectivas". Ficha de Cátedra, "Técnicas de evaluación y exploración diagnóstica II", Carrera de Psicología, UCES, repositorio online http://dspace.uces.edu.ar:8180/ xmlui/browse?order $=$ ASC\&rpp $=20 \&$ sort by $=-1 \&$ value $=$ Caride + de + Mizes $\% 2 C+$ Mar\%C3\%ADa+R.\&etal=-1\&offset=0\&type $=$ author

Caride, M. (2008) Indicadores de malestar social a través del Test de Relaciones Objetales (de H. Phillipson), XV Jornadas de Investigación. Cuarto encuentro de investigadores en psicología del Mercosur, 7 al 9 de Agosto de 2008. Recuperado de http://dspace.uces, edu.ar:8180/xmlui/handle/123456789/1527

Carney, S. (1992). Draw a person in the rain: A comparison of levels of stress and depression among adolescents. ETD Collection for Pace University, Paper Al9305306. Resumen recuperado de http://digitalcommons. pace.edu/dissertations/AAI9305306

Casullo, M. (1998). El Test Gráfico del Dibujo de la Figura Humana. Normas regionales. Buenos Aires: Guadalupe.

de Souza, L. \& Guerrero, L. (2005). Investigación sobre Cambio Psíquico. Estudio longitudinal en procesos psicoterapéuticos. En A. Muniz (Comp.), Diagnósticos e Intervenciones. Enfoques Teóricos, Técnicos y Clínicos en la Práctica Psicológica. Tomo 3 (pp. 145-155). Montevideo: Editorial Psicolibros.

Fatelevich, M., Biasella, R., Castillo, C., Coppola, M., Luque, A., Maglio, N., ... \& Schwartz, L. (2008). Violencia de género: Descripción y resultados de una investigación sobre mujeres golpeadas. Revista de Psicología (La Plata), 10, 237-247. Recuperado de http://www.memoria.fahce.unlp.edu.ar/art revistas/pr.4410/pr.4410.pdf

Freud, S. (1915). Pulsiones y destinos de pulsión (Vol. 14). Buenos Aires: Amorrortu Editores.

Freud, S. (1920). Más allá del principio del placer (Vol. 18). Buenos Aires: Amorrortu Editores.

Freud, S. (1926). Inhibición, síntoma y angustia (Vol. 20). Buenos Aires: Amorrortu Editores.

Goodenough, J. (1983). El dibujo infantil (3ra ed.). Madrid: Morata.

Grassano de Piccolo, E. (1977). Indicadores psicopatológicos en técnicas proyectivas. Buenos Aires: Nueva Visión.

Grinberg, L. (1976). Psicoanálisis: aportes teóricos y clínicos. Barcelona: Alex Editor.

Groupe, $\mu$. (1993). Tratado del signo visual. Para una retórica de la imagen. Madrid: Cátedra.

Hammer, E. (1969). Test proyectivos gráficos. Barcelona: Editorial Paidós.

Koppitz, E. (1975). El dibujo de la figura humana en los niños. Buenos Aires: Guadalupe.

Kris, E. (1952). Psicoanálisis del arte y del artista. Buenos Aires: Paidós.

Lacan, J. (1997). El yo en la teoría de Freud y en la técnica psicoanalítica. Buenos Aires: Paidós.

Lacan, J. (1958). De una cuestión preliminar a todo tratamiento posible de la psicosis. Escritos, 2, 513-564.

Liberman, D. (1972). Lingüística, interacción comunicativa y proceso psicoanalítico. Buenos Aires: Galerna-Nueva Visión.

Machover, K. (1949). Personality projection in the drawings of the human figure. Springfield, IL: Thomas.

Maldavsky, D. (2004). La investigación psicoanalítica del lenguaje: algoritmo David Liberman. Buenos Aires: Lugar.

Maldavsky, D. (2013). ADL. Algoritmo David Liberman: Un instrumento para la evaluación de los deseos y las defensas en el discurso. Buenos Aires: Paidós.

Maldavsky, D. (2014a). Un método para la investigación del deseo y la defensa en el discurso: algoritmo David Liberman (ADL). Revista Linguagem \& Ensino, 17(1), 151-169. Recuperado de http://www.rle.ucpel.tche.br/ index.php/rle/article/view/933

Maldavsky, D. (2014b). Método de estudio de los deseos y las defensas en el componente icónico del signo visual (ADL-SV). Revista Linguagem \& Ensino, 17(2), 471505. Recuperado de http://www.rle.ucpel.tche.br/ index.php/rle/article/view/1090

Maldavsky, D., Aguirre, A., Álvarez, L., Bodni, O., Britti, M., Bu- 
Un nuevo diseño para el análisis de la respuesta gráfica al Test de Persona Bajo la Lluvia, APLICADO EN PSICOLOGÍA LABORAL: DETECCIÓN DE LOS RECURSOS FRENTE AL TRAUMA

ceta, C., ... \& Varela, R. (2007). La intersubjetividad en la clínica psicoanalítica. Investigación sistemática con el algoritmo David Liberman (ADL). Buenos Aires: Lugar.

McDougall, J. (1989). Teatros del cuerpo. Madrid: Julián Yébenes.

Nudel, C. (2014). Abuso sexual intrafamiliar: el dibujo conjunto como medio de evaluación. Lo icónico y lo plástico en el gráfico. Subjetividad y procesos cognitivos, 18(1), 245-273. Recuperado de http://ref.scielo.org/56xc2c

Perez Zambón, S. (2014). Aplicación de la metodología del algoritmo David Liberman (ADL) al análisis del componente icónico y de los relatos correspondientes al test de Persona Bajo la Lluvia (PBLL). Revista Linguagem \& Ensino, 17(2), 507-524. Recuperado de http://www. rle.ucpel.tche.br/index.php/rle/article/view/1091/

Perez Zambón, S. (2015). El test de persona bajo la lluvia: Una nueva perspectiva de análisis. Subjetividad y procesos cognitivos, 19(1), 200-227. Recuperado de http:// ref.scielo.org/2wkt55

Piccone, A. \& Passalacqua, A. (2010). Algunas observaciones para evaluar las funciones yoicas de realidad con la aplicación de una técnica gráfica. Anuario de Investigaciones, 17, 185-194. Recuperado de http://ref.scielo. org/v7xbj9

Pool, A. (2006). Análisis desde el modelo traumatogénico de los indicadores gráficos asociados a agresiones sexuales infantiles en la Prueba Persona Bajo la Lluvia. Psykhe, 15(1), 45-55. doi:10.4067/S071822282006000100004

Portuondo, J. (1973). Test proyectivo de Karen Machover. Madrid: Biblioteca Nueva.

Prichard, B. (1998). The utillity of the Draw-A-Person in the Rain as a measure of stress and concomitant depressive and anxiety symptoms in elementary school children (Tesis doctoral inédita). National Luis University, Chicago.

Querol, S. \& Alcañiz, S. (2001). Selección de personal: aplicación del test de la persona bajo la lluvia y análisis grafológico del relato. Buenos Aires: Lugar.
Querol, S. \& Chaves Paz, M. (1997). La persona bajo la lluvia. Buenos Aires: JVE Psique.

Rapaport D. (1953). Sobre la teoría psicoanalítica de los afectos. En M. Gill \& D. Rapaport (Eds.), Aportaciones a la teoría y técnica psicoanalítica (pp. 118-169). México: Pax.

Rossi, A. (1997). The draw-a-person-in-the-rain technique: a study to determine its use as an informative, adjunct assessment tool for direct practice social workers (Tesis de maestría inédita). Southern Connecticut State University, Connecticut.

Siquier de Ocampo, M. \& García Arzeno, M. (1974). El proceso psicodiagnóstico. En M. Siquier de Ocampo, M. García Arzeno \& E. Grassano (Comp.), Las técnicas proyectivas y el proceso psicodiagnóstico (pp. 11-20). Buenos Aires: Nueva Visión.

Sneiderman, S. (octubre de 1998). Algunos indicadores psicopatológicos a la luz de las técnicas de exploración en la clínica de los procesos tóxicos, Psicopatología. Actualizando Psicodiagnóstico. Trabajo presentado en el II Congreso Nacional de Psicodiagnóstico, IX Jornadas Nacionales de ADEIP, Santa Fe.

Taylor, P. (1977). An investigation of the utility of the Draw-APerson-In-The-Rain for assessment of stress and the prediction of achievement in college students (Doctoral dissertation). Recuperada de ProQuest Information \& Learning.

Verinis, J., Lichtenberg, E. \& Henrich, L. (1974). The drawA-person in the rain technique: Its relationship to diagnostic category and other personality indicators. Journal of Clinical Psychology, 30(3), 407-414. doi:10.1002/1097-4679(197407)30:3<407::AID-JCLP2270300358>3.0.CO;2-6

Willis, L., Joy, S. \& Kaiser, D. (2010). Draw-a-Person-in-theRain as an assessment of stress and coping resources. The Arts in Psychotherapy, 37(3), 233-239. doi:10.1016/j.aip.2010.04.009 